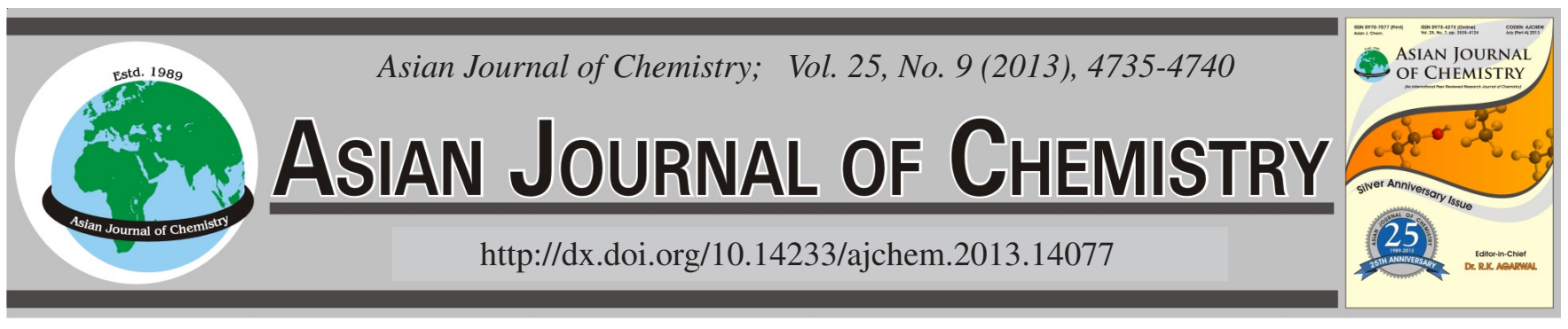

\title{
DFT Calculations of Vibrational Frequencies of Aluminum and Phosphorous Doped-Carbon Clusters
}

\author{
Maqsood Ahmad Malik ${ }^{1}$, Firdosa Nabi ${ }^{2, *}$, Christopher G. Jesudason ${ }^{2}$ and Shaeel Ahmed Al-Thabaiti ${ }^{1}$
}

${ }^{1}$ Department of Chemistry, Faculty of Science, King Abdulaziz University, P.O. Box 80203, Jeddah 21413, Saudi Arabia

${ }^{2}$ Department of Chemistry, University of Malaya, Kuala Lumpur 50603, Malaysia

*Corresponding author: Tel: +60 10 5444912; E-mail: firdosachem@gmail.com

(Received: 21 May 2012;

Accepted: 4 March 2013)

AJC-13060

We have designed numerous models of aluminum $\left(\mathrm{C}_{\mathrm{n}} \mathrm{Al} \mathrm{l}_{\mathrm{m}}\right)$ and phosphorus $\left(\mathrm{C}_{\mathrm{n}} \mathrm{P}_{\mathrm{m}}\right)$ doped carbon clusters $(\mathrm{n}=3$ and $\mathrm{m}=4)$. The geometry
optimization, bond length and calculation of vibrational frequency were carried out in each case by DFT in local density approximation.
We also try other widely used functionals but local density approximation functional works well in our study. Numerous cluster structures
of aluminum and phosphorus were prepared because of their different bonding preference. In addition, the total energies of the $\mathrm{C}_{\mathrm{n}} \mathrm{Al}_{\mathrm{m}}$ and
$\mathrm{C}_{\mathrm{n}} \mathrm{P}_{\mathrm{m}}$ clusters were also discussed.
Key Words: DFT, Binary clusters, Vibrational frequencies, Energies.

\section{INTRODUCTION}

Atomic clusters have become the field of interest from last few years due to their significant role as bridge between microscopic atoms and macroscopic condensed matter. Doping with molecular species adds further multidimensionality to the cluster structure and property variations, which can be useful for practical applications such as catalysis, new (nanostructured) materials, or molecular devices ${ }^{1}$. The electronic structure calculation, based on density functional theory (DFT) and the local density approximation (LDA) are the reliable and computationally tractable tool in materials science ${ }^{2-5}$. A deep investigation of the properties of the doped carbon clusters is desirable, because the hetero-atoms are expected to play special role in their physical and chemical properties.

Zhan and Iwata ${ }^{6}$ optimized the geometric structures of $\mathrm{C}_{\mathrm{n}} \mathrm{P}^{-}(\mathrm{n}=1-7)$ using ab initio calculations. Pascoli and Lavendy proposed structures of carbon and phosphorus clusters $\left(\mathrm{C}_{\mathrm{n}} \mathrm{P}\right.$, $\mathrm{C}_{\mathrm{n}} \mathrm{P}^{-}, \mathrm{C}_{\mathrm{n}} \mathrm{P}^{+}(\mathrm{n}=1-7)$ and $\left.\mathrm{C}_{\mathrm{n}} \mathrm{P}_{\mathrm{p}}^{+}(\mathrm{n}+\mathrm{p}=3-6)\right)$ based on data collected in DFT calculations ${ }^{7-9}$. Liu and his coworkers carried out calculations on linear $\mathrm{C}_{\mathrm{n}} \mathrm{P}^{-}(\mathrm{n}=1-11)$ by using the HF method $^{10,11}$. Fisher et al. ${ }^{12}$ conducted density functional calculations on structures of some carbon phosphide anions. $\mathrm{C}_{n} \mathrm{P}_{2}$ ( $n=3-9)$ clusters have been analyzed theoretically by DFT calculations $^{13}$, whereas various geometries of $\mathrm{C}_{3} \mathrm{P}$ and $\mathrm{C}_{3} \mathrm{PC}$ have been investigated by other $a b$ initio approaches ${ }^{14}$. Various studies have been reported on aluminum clusters like $\mathrm{C}_{2} \mathrm{Al}$ and $\mathrm{C}_{2} \mathrm{Al}_{2}$ in order to relate the geometry with stability ${ }^{15,16}$.
Recently, Naumkin ${ }^{1}$ studied the stable geometries, which correspond to the flat-structural motives of $\mathrm{CAl}_{3}$ units. His content of "stability" refers to the energies among a predetermined set of clusters. The resemblances of $\mathrm{C}_{2} \mathrm{Al}_{3}$ with $\mathrm{C}_{2} \mathrm{Al}_{2}$ have been reported but many apparent questions regarding the geometries of $\mathrm{C}_{\mathrm{n}} \mathrm{Al}_{\mathrm{m}}$ remain unanswered ${ }^{17}$. To the best of our knowledge no work on the theoretical study of phosphorous and aluminum-doped carbon clusters $(n=3$ and $m=4)$ has been reported. Since the physical and chemical properties of a small metal cluster rely on its atomic structure, determining the lowest-energy structures of clusters is a fundamental step in understanding and utilizing their properties. In view of these facts, the present study has been undertaken to have a better understanding of the physical and chemical properties in phosphorous and aluminum-doped carbon clusters.

\section{COMPUTATIONAL METHOD}

DFT is a commonly used, reliable and accurate approach in searching for the lowest-energy structures of $\mathrm{C}_{\mathrm{n}} \mathrm{Al}_{\mathrm{m}}$ and $\mathrm{C}_{\mathrm{n}} \mathrm{P}_{\mathrm{m}}$ doped carbon clusters. When the cluster becomes larger, i.e., $\mathrm{n}$ increases, the number of energy minima for a given cluster size increases rapidly. For this reason, an impartial search for the lowest-energy structure is extremely vital. DFT is used to obtain the solution of the Schrödinger equation within the local-density approximation (LDA). The Amsterdam densityfunctional theory (ADF) package using the double zeta wave functions was utilized in this study. The theory on determing the vibrational frequencies has been well described earlier ${ }^{18}$. 
All possible clusters were made starting from the simplest one with only two atoms. For this cluster, the electronic energy is calculated as a function of distance between atoms and hence the lowest energy distances can be determined. The lowestenergy configuration is considered to be the stable one and the others with maximum energy are considered to be unstable and discarded. In a second step, the vibrational frequencies were calculated for the stable structures. In short, the optimization of the electronic energy determines the structure and the vibrational frequencies were always obtained for the optimized structure. We build all of the possible clusters and retain only the stable ones. All structures of clusters were fully optimized freely and only those structures that had a symmetry property were retained in this study. The reason is that we propose the larger structures may be built up from there elementary units which possess some symmetrical property.

\section{RESULTS AND DISCUSSION}

The present work deals with the theoretical study of aluminum and phosphorous-doped carbon clusters. We present the vibrational frequencies, zero point energies and total energies of the various aluminum and phosphorous-doped carbon clusters. These clusters are very interesting from the theoretical point of view because of the geometry difference between two different pairs $\left(\mathrm{C}_{n} \mathrm{Al}_{\mathrm{m}}\right.$ and $\left.\mathrm{C}_{\mathrm{n}} \mathrm{P}_{\mathrm{m}}\right)$ of heterodoped carbon clusters. Both aluminum (group III) and phosphorus (group V) are adjacent to carbon (group IV) in periodic table and relative to carbon, aluminum can be considered to be electron poor and phosphorus electron rich. Hence, it is interesting to make comparisons between the two types because of their different group properties. Ball and stick models are used to represent clusters of $\mathrm{C}_{\mathrm{n}} \mathrm{Al}_{\mathrm{m}}$ and $\mathrm{C}_{\mathrm{n}} \mathrm{P}_{\mathrm{m}}$ (Figs. 1 and 2). Bigger balls represent the aluminum and phosphorus atoms and smaller ones denote carbon atoms. The geometries of most of the clusters depicted here has never been reported before.

\section{Geometries of $\mathrm{C}_{\mathrm{n}} \mathrm{Al}_{\mathrm{m}}$ and $\mathrm{C}_{\mathrm{n}} \mathrm{P}_{\mathrm{m}}$ systems}

$\mathbf{C A l}_{n}$ clusters $\left(\mathbf{C A l}, \mathbf{C A l}_{2}, \mathbf{C A l}_{3}\right.$ and $\left.\mathbf{C A l}_{4}\right)$ : We made the diatomic molecule $\mathrm{CAl}$ [Fig. 1(i)] and optimized the structure for the minimum energy. Adding a single $\mathrm{Al}$ atom to $\mathrm{C}$ can produce a linear isomer, which could formally be associated with the $\mathrm{C}$-Al bond length of $1.964 \AA$ and the appreciable lowest vibrational frequency of $750.5 \mathrm{~cm}^{-1}$. In the linear model $\mathrm{CAl}_{2}$ linear [Fig. 1(ii)], the carbon atom is kept in the center and two aluminum atoms are located on each side of $\mathrm{C}$ so that the $\mathrm{Al}-\mathrm{C}-\mathrm{Al}$ atoms are aligned in a straight line. The bond length of $\mathrm{C}-\mathrm{Al}$ is found to be $1.818 \AA$. The results of the vibrational frequencies of significant intensity allow us to consider the following isomers. The $\mathrm{CAl}_{3}$ (triangular) model [Fig. 1(iii)] is formed by three atoms of $\mathrm{Al}$ which sit on three corners of a triangle and $\mathrm{C}$ sits in the center of the triangle. The $\mathrm{CAl}$ bond length is found to be $1.917 \AA$ and in square model $\mathrm{CAl}_{4}$ [Fig. 1(iv)] in which the four $\mathrm{Al}$ atoms sit on the corners of a square and one $\mathrm{C}$ is located in the center of the square. The $\mathrm{CAl}$ bond length is $2.471 \AA$. All the vibrational frequencies are given in Table-1.

$\mathbf{C}_{\mathbf{n}} \mathrm{Al}$ clusters $\left(\mathbf{C}_{2} \mathrm{Al}, \mathbf{C}_{3} \mathrm{Al}\right)$ : Adding a single $\mathrm{Al}$ atom to $\mathrm{C}_{2}$ molecule can produce a linear isomer $\mathrm{C}_{2} \mathrm{Al}$ [Fig. 1(v)] with one $\mathrm{Al}$ in the center and two $\mathrm{C}$ on two corners. The $\mathrm{C}$-Al bond length is found to be $2.200 \AA$ and in the triangular model $\mathrm{C}_{2} \mathrm{Al}$ [Fig. 1(vi)] the CAl distance is found to be $2.045 \AA$ and C-C distance is $1.271 \AA$. In the $\mathrm{C}_{3} \mathrm{Al}$ pyramidal model, [Fig. 1(vii)] the three carbon atoms form a triangle and the $\mathrm{Al}$ atom sits at the top position. The $\mathrm{C}-\mathrm{Al}$ bond distance is $1.975 \AA$ and the $\mathrm{C}-\mathrm{C}$ bond length is $1.499 \AA$ and in the $\mathrm{C}_{3} \mathrm{Al}$ triangular model [Fig. 1(viii)] the three $\mathrm{C}$ atoms form a triangle and one $\mathrm{Al}$ atom sits at the center. In this case the $\mathrm{C}$ - $\mathrm{Al}$ bond length is $1.953 \AA$. All the calculated vibrational frequencies are represented in Table- 1 .

$\mathrm{C}_{\mathrm{n}} \mathrm{Al}_{\mathrm{m}}$ clusters $\left(\mathrm{C}_{2} \mathrm{Al}_{2}, \mathrm{C}_{2} \mathrm{Al}_{3}, \mathrm{C}_{3} \mathrm{Al}_{2}\right.$ and $\left.\mathrm{C}_{3} \mathrm{Al}_{3}\right)$ : Figs. 1 (ix) to 1 (xiv) list, respectively, the different geometries of the cluster systems. Optimized bond lengths of $\mathrm{C}_{\mathrm{n}} \mathrm{Al}_{\mathrm{m}}$ clusters

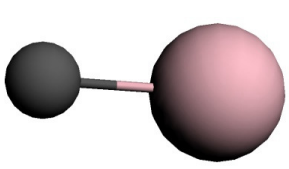

(i)

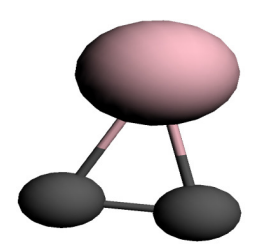

(vi)

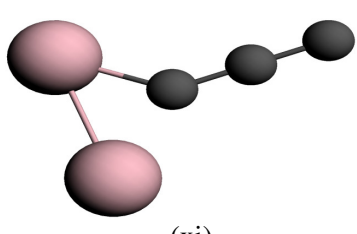

(xi)

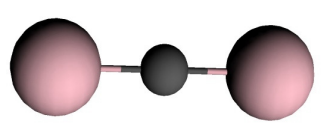

(ii)

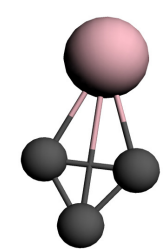

(vii)

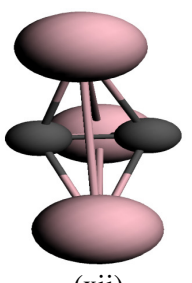

(xii)

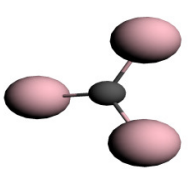

(iii)

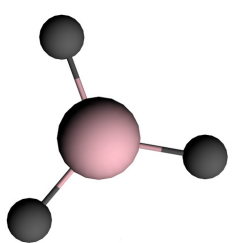

(viii)

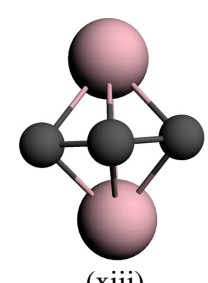

(xiii)

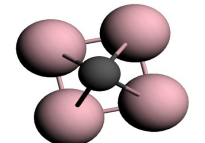

(iv)

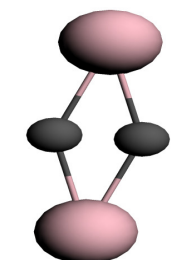

(ix)

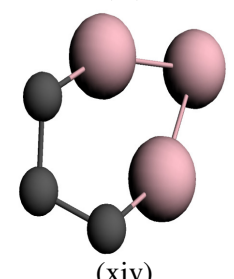

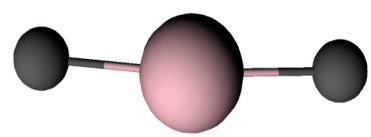

(v)

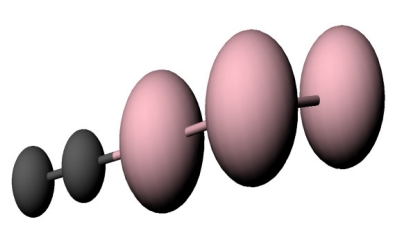

(x)

Fig. 1. Qualitative geometry of $\mathrm{C}_{\mathrm{n}} \mathrm{Al}_{\mathrm{m}}$ clusters (i) to (xiv) shown by ball and stick models without electronic density contours 
(i)

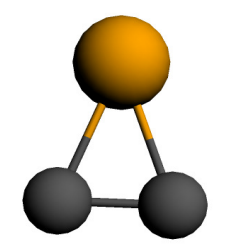

(vi)

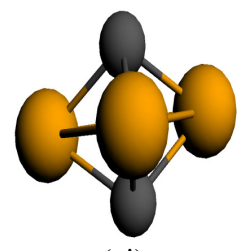

(xi)

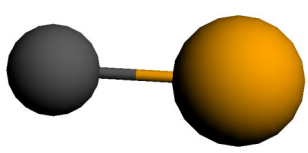

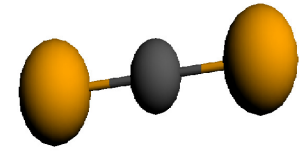

(ii)

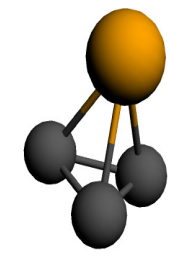

(vii)

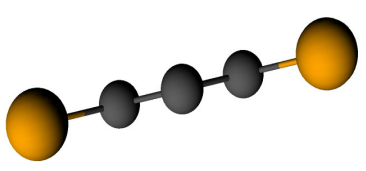

(xii)

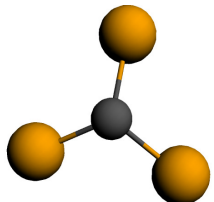

(iii)

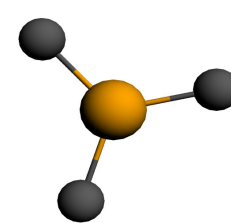

(viii)

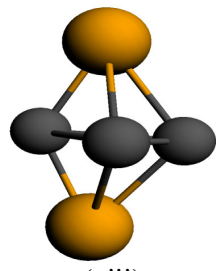

(xiii)

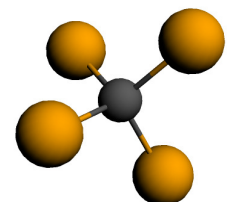

(iv)

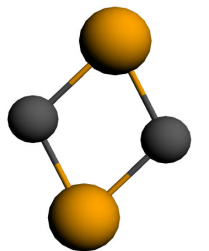

(ix)

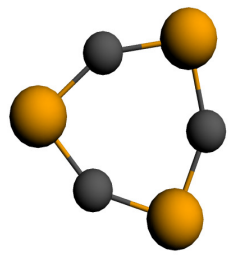

(xiv)

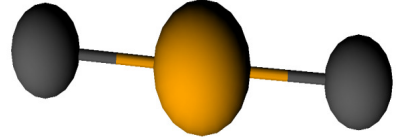

(v)

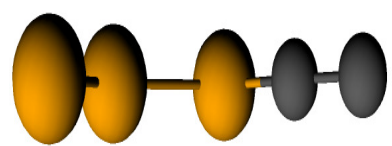

(x)
Fig. 2. Qualitative geometry of clusters (i) to (xiv) shown by stick-ball models without electronic structure

\begin{tabular}{|c|c|c|c|c|}
\hline \multicolumn{5}{|c|}{$\begin{array}{l}\text { VIBRATIONAL FREQUENCIES OF VARIOUS CLUSTERS } \\
\text { CALCULATED FROM THE FIRST ORDER PRINCIPLES }\end{array}$} \\
\hline $\begin{array}{l}\text { S. } \\
\text { No. }\end{array}$ & Cluster & $\begin{array}{l}\text { Frequency } \\
\left(\mathrm{cm}^{-1}\right)\end{array}$ & $\begin{array}{l}\text { Intensity } \\
(\mathrm{km} / \mathrm{mol})\end{array}$ & Degeneracy \\
\hline \multicolumn{5}{|c|}{$\mathrm{CAl}_{\mathrm{n}}$ clusters } \\
\hline 1 & $\mathrm{CAl}_{2}$ (linear) & 82.8 & 97.9 & 1 \\
\hline 2 & $\mathrm{CAl}_{2}$ (linear) & 454.9 & 0.01 & 1 \\
\hline 3 & $\mathrm{CAl}_{2}$ (linear) & 1043.9 & 36.5 & 1 \\
\hline 4 & $\mathrm{CAl}_{3}$ (triangular) & 449.3 & 2.05 & 2 \\
\hline 5 & $\mathrm{CAl}_{3}$ (triangular) & 210.3 & 15.21 & 1 \\
\hline 6 & $\mathrm{CAl}_{3}$ (triangular) & 395.5 & 0.01 & 1 \\
\hline 7 & $\mathrm{CAl}_{3}$ (triangular) & 820.3 & 361.9 & 2 \\
\hline 8 & $\mathrm{CAl}_{4}$ (square) & 103.2 & 0.01 & 2 \\
\hline 9 & $\mathrm{CAl}_{4}$ (square) & 184.1 & 8.7 & 3 \\
\hline 10 & $\mathrm{CAl}_{4}$ (square) & 330.2 & 0.01 & 1 \\
\hline 11 & $\mathrm{CAl}_{4}$ (square) & 586.7 & 250.0 & 3 \\
\hline \multicolumn{5}{|c|}{$\mathrm{C}_{\mathrm{n}} \mathrm{Al}$ clusters } \\
\hline 1 & $\mathrm{C}_{2} \mathrm{Al}$ (linear) & 192.0 & 30.5 & 1 \\
\hline 2 & $\mathrm{C}_{2} \mathrm{Al}$ (linear) & 325.3 & 0.1 & 1 \\
\hline 3 & $\mathrm{C}_{2} \mathrm{Al}$ (linear) & 474.5 & 44.5 & 1 \\
\hline 4 & $\mathrm{C}_{2} \mathrm{Al}$ (triangular) & 149.3 & 8.1 & 1 \\
\hline 5 & $\mathrm{C}_{2} \mathrm{Al}$ (triangular) & 579.1 & 174.8 & 1 \\
\hline 6 & $\mathrm{C}_{2} \mathrm{Al}$ (triangular) & 1785.1 & 15.4 & 1 \\
\hline 7 & $\mathrm{C}_{3} \mathrm{Al}$ (pyramidal) & 261.7 & 0.01 & 1 \\
\hline 8 & $\mathrm{C}_{3} \mathrm{Al}$ (pyramidal) & 266.4 & 25.6 & 1 \\
\hline 9 & $\mathrm{C}_{3} \mathrm{Al}$ (pyramidal) & 433.8 & 137.9 & 1 \\
\hline 10 & $\mathrm{C}_{3} \mathrm{Al}$ (pyramidal) & 790.1 & 25.1 & 1 \\
\hline 11 & $\mathrm{C}_{3} \mathrm{Al}$ (pyramidal) & 1256.9 & 0.33 & 1 \\
\hline 12 & $\mathrm{C}_{3} \mathrm{Al}$ (pyramidal) & 1462.2 & 27.0 & 1 \\
\hline 13 & $\mathrm{C}_{3} \mathrm{Al}$ (triangular) & 422.6 & 22.2 & 1 \\
\hline 14 & $\mathrm{C}_{3} \mathrm{Al}$ (triangular) & 526.6 & 0.01 & 1 \\
\hline 15 & $\mathrm{C}_{3} \mathrm{Al}$ (triangular) & 723.7 & 15.5 & 2 \\
\hline \multicolumn{5}{|c|}{$\mathrm{C}_{\mathrm{n}} \mathrm{Al}_{\mathrm{m}}$ clusters } \\
\hline 1 & $\mathrm{C}_{2} \mathrm{Al}_{2}$ (rectangular) & 280.2 & 0.01 & 1 \\
\hline 2 & $\mathrm{C}_{2} \mathrm{Al}_{2}$ (rectangular) & 430.8 & 805.4 & 1 \\
\hline 3 & $\mathrm{C}_{2} \mathrm{Al}_{2}$ (rectangular) & 1784.6 & 0.01 & 1 \\
\hline 4 & $\mathrm{C}_{2} \mathrm{Al}_{3}$ (linear) & 88.7 & 11.8 & 1 \\
\hline 5 & $\mathrm{C}_{2} \mathrm{Al}_{3}$ (linear) & 196.7 & 4.7 & 1 \\
\hline 6 & $\mathrm{C}_{2} \mathrm{Al}_{3}$ (linear) & 317.0 & 18.6 & 1 \\
\hline
\end{tabular}

\begin{tabular}{|c|c|c|c|c|}
\hline 7 & $\mathrm{C}_{2} \mathrm{Al}_{3}$ (linear) & 347.7 & 32.1 & 1 \\
\hline 8 & $\mathrm{C}_{2} \mathrm{Al}_{3}$ (linear) & 467.7 & 0.17 & 1 \\
\hline 9 & $\mathrm{C}_{2} \mathrm{Al}_{3}$ (linear) & 656.6 & 6.8 & 1 \\
\hline 10 & $\mathrm{C}_{2} \mathrm{Al}_{3}$ (linear) & 1899.6 & 536.4 & 1 \\
\hline 11 & $\mathrm{C}_{2} \mathrm{Al}_{3}$ (bipyramidal) & 249.1 & 2.4 & 2 \\
\hline 12 & $\mathrm{C}_{2} \mathrm{Al}_{3}$ (bipyramidal) & 380.2 & 0.01 & 1 \\
\hline 13 & $\mathrm{C}_{2} \mathrm{Al}_{3}$ (bipyramidal) & 396.1 & 0.01 & 2 \\
\hline 14 & $\mathrm{C}_{2} \mathrm{Al}_{3}$ (bipyramidal) & 487.2 & 62.0 & 1 \\
\hline 15 & $\mathrm{C}_{2} \mathrm{Al}_{3}$ (bipyramidal) & 570.8 & 83.4 & 2 \\
\hline 16 & $\mathrm{C}_{2} \mathrm{Al}_{3}$ (bipyramidal) & 678.5 & 0.01 & 1 \\
\hline 17 & $\mathrm{C}_{3} \mathrm{Al}_{2}$ (linear) & 101.2 & 2.5 & 1 \\
\hline 18 & $\mathrm{C}_{3} \mathrm{Al}_{2}$ (linear) & 169.2 & 0.7 & 1 \\
\hline 19 & $\mathrm{C}_{3} \mathrm{Al}_{2}$ (linear) & 172.0 & 8.1 & 1 \\
\hline 20 & $\mathrm{C}_{3} \mathrm{Al}_{2}$ (linear) & 290.4 & 8.0 & 1 \\
\hline 21 & $\mathrm{C}_{3} \mathrm{Al}_{2}$ (linear) & 434.6 & 5.2 & 1 \\
\hline 22 & $\mathrm{C}_{3} \mathrm{Al}_{2}$ (linear) & 439.4 & 41.4 & 1 \\
\hline 23 & $\mathrm{C}_{3} \mathrm{Al}_{2}$ (linear) & 620.5 & 219.7 & 1 \\
\hline 24 & $\mathrm{C}_{3} \mathrm{Al}_{2}$ (linear) & 1186.8 & 0.04 & 1 \\
\hline 25 & $\mathrm{C}_{3} \mathrm{Al}_{2}$ (linear) & 1878.3 & 657.5 & 1 \\
\hline 26 & $\mathrm{C}_{3} \mathrm{Al}_{2}$ (bipyramidal) & 280.0 & 53.3 & 1 \\
\hline 27 & $\mathrm{C}_{3} \mathrm{Al}_{2}$ (bipyramidal) & 409.2 & 8.5 & 1 \\
\hline 28 & $\mathrm{C}_{3} \mathrm{Al}_{2}$ (bipyramidal) & 417.1 & 9.9 & 1 \\
\hline 29 & $\mathrm{C}_{3} \mathrm{Al}_{2}$ (bipyramidal) & 541.5 & 37.8 & 1 \\
\hline 30 & $\mathrm{C}_{3} \mathrm{Al}_{2}$ (bipyramidal) & 546.4 & 97.1 & 1 \\
\hline 31 & $\mathrm{C}_{3} \mathrm{Al}_{2}$ (bipyramidal) & 725.7 & 1.5 & 1 \\
\hline 32 & $\mathrm{C}_{3} \mathrm{Al}_{2}$ (bipyramidal) & 989.4 & 37.4 & 1 \\
\hline 33 & $\mathrm{C}_{3} \mathrm{Al}_{3}$ (ring) & 82.0 & 33.5 & 1 \\
\hline 34 & $\mathrm{C}_{3} \mathrm{Al}_{3}$ (ring) & 126.1 & 0.05 & 1 \\
\hline 35 & $\mathrm{C}_{3} \mathrm{Al}_{3}$ (ring) & 161.4 & 64.9 & 1 \\
\hline 36 & $\mathrm{C}_{3} \mathrm{Al}_{3}$ (ring) & 169.1 & 1.4 & 1 \\
\hline 37 & $\mathrm{C}_{3} \mathrm{Al}_{3}$ (ring) & 217.2 & 11.3 & 1 \\
\hline 38 & $\mathrm{C}_{3} \mathrm{Al}_{3}$ (ring) & 298.4 & 1.2 & 1 \\
\hline 39 & $\mathrm{C}_{3} \mathrm{Al}_{3}$ (ring) & 346.7 & 3.0 & 1 \\
\hline 40 & $\mathrm{C}_{3} \mathrm{Al}_{3}$ (ring) & 428.1 & 0.2 & 1 \\
\hline 41 & $\mathrm{C}_{3} \mathrm{Al}_{3}$ (ring) & 445.6 & 14.2 & 1 \\
\hline 42 & $\mathrm{C}_{3} \mathrm{Al}_{3}$ (ring) & 493.3 & 143.7 & 1 \\
\hline 43 & $\mathrm{C}_{3} \mathrm{Al}_{3}$ (ring) & 571.6 & 26.5 & 1 \\
\hline
\end{tabular}

and the $\mathrm{C}_{\mathrm{n}}$ and $\mathrm{Al}_{\mathrm{m}}$ alternation effect are also exhibited for the various clusters. In the $\mathrm{C}_{2} \mathrm{Al}_{2}$ rectangular model [Fig. 1(ix)] 
the atoms are alternately arranged with the bond length 2.308 $\AA$. The $\mathrm{C}_{2} \mathrm{Al}_{3}$ linear model [Fig. 1(x)] is obtained with two $\mathrm{Al}$ atoms and one $\mathrm{C}$ atom which lies in the center and one $\mathrm{C}$ and $\mathrm{Al}$ at the ends. The Al-Al bond length is $2.586 \AA$ and C-C distance observed is $1.268 \AA$. The $\mathrm{C}_{3} \mathrm{Al}_{2}$ [Fig. 1(xi)] linear model is represented by two $\mathrm{C}$ and $\mathrm{Al}$ atoms which lie at the center and $\mathrm{C}$ and $\mathrm{Al}$ at the ends. The $\mathrm{Al}-\mathrm{Al}$ distance is 2.6192 $\AA$ and C-C distance is $1.2952 \AA$ at the ends. On optimization of the linear isomer, the bent geometry is displayed with no linearity in structure. The bipyramidal model [Fig. 1(xii)] $\mathrm{C}_{2} \mathrm{Al}_{3}$ is formed by three aluminum atoms, which form a triangle and one $\mathrm{C}$ atom sits on the top position and another in the bottom position. The $\mathrm{CAl}$ distance is found to be $2.364 \AA$ and the $\mathrm{Al}$-Al distance $3.934 \AA$. Similarly, in the $\mathrm{C}_{3} \mathrm{Al}_{2}$ bipyramidal structure [Fig. 1(xiii)] the three carbon atoms form a triangle and one $\mathrm{Al}$ atom is at the top position and another at the bottom position. The $\mathrm{CAl}$ distance is $2.295 \AA$ and $\mathrm{Al}-\mathrm{Al}$ distance is $3.196 \AA$. The $\mathrm{C}_{3} \mathrm{Al}_{3}$ [Fig. 1(xiv)] model is a hexagonal ring with three sites occupied by $\mathrm{C}$ on one side of the ring and three $\mathrm{Al}$ atoms on the other side. All the vibrational frequencies calculated from the first principles ${ }^{9}$ are given in Table- 1 .

$\mathbf{C P}_{\mathbf{n}}$ clusters $\left(\mathbf{C P}, \mathbf{C P}_{\mathbf{2}}, \mathbf{C P}_{\mathbf{3}}\right.$ and $\left.\mathbf{C P}_{\mathbf{4}}\right)$ : We now consider $\mathrm{CP}_{\mathrm{n}}$ clusters and the corresponding geometries are displayed in Fig. 2. In each case, the geometry is optimized starting from several initial configurations obtained by adding the various atoms. Using the same principles as previously described we make the diatomic molecule CP [Fig. 2(i)] and optimized the structure for minimum energy. This calculation gives the $\mathrm{CP}$ bond length of $1.613 \AA$ with vibrational frequency calculated as $1165.8 \mathrm{~cm}^{-1}$. Another $\mathrm{CP}_{2}$ linear [Fig. 2(ii)] model is obtained by adding two phosphorus atoms on each side of $\mathrm{C}$, which is kept at the center so that P-C-P atoms are aligned in a straight line. The bond length of C-P is found to be $1.664 \AA$. Addition of three atoms of $\mathrm{P}$ to $\mathrm{C}$ which occupies the center and $\mathrm{P}$ which sit on three corners of a triangle results in a triangular $\mathrm{CP}_{3}$ [Fig. 2(iii)] model. The $\mathrm{CP}$ bond length is found to be $1.773 \AA$. Another model of $\mathrm{CP}_{4}$ [Fig. 2(iv)] is a square in which the four $\mathrm{P}$ atoms are on the corners of a square and one $\mathrm{C}$ located in the center of the square. The $\mathrm{CP}$ bond length is $1.818 \AA$. All the vibrational frequencies are given in Table-2.

\begin{tabular}{|c|c|c|c|c|}
\hline \multicolumn{5}{|c|}{$\begin{array}{c}\text { TABLE-2 } \\
\text { VIBRATIONAL FREQUENCIES OF VARIOUS CLUSTERS } \\
\text { CALCULATED FROM THE FIRST ORDER PRINCIPLES }\end{array}$} \\
\hline $\begin{array}{l}\text { S. } \\
\text { No. }\end{array}$ & Cluster & $\begin{array}{l}\text { Frequency } \\
\left(\mathrm{cm}^{-1}\right)\end{array}$ & $\begin{array}{l}\text { Intensity } \\
(\mathrm{km} / \mathrm{mol})\end{array}$ & Degeneracy \\
\hline \multicolumn{5}{|c|}{$\mathrm{CP}_{\mathrm{n}}$ clusters } \\
\hline 1 & $\mathrm{CP}_{2}$ (linear) & 475.3 & 41.3 & 2 \\
\hline 2 & $\mathrm{CP}_{2}$ (linear) & 545.1 & 0.5 & 1 \\
\hline 3 & $\mathrm{CP}_{2}$ (linear) & 1401.3 & 198.7 & 1 \\
\hline 4 & $\mathrm{CP}_{3}$ (triangular) & 222.8 & 7.17 & 2 \\
\hline 5 & $\mathrm{CP}_{3}$ (triangular) & 441.8 & 0.3 & 1 \\
\hline 6 & $\mathrm{CP}_{3}$ (triangular) & 659.8 & 27.7 & 1 \\
\hline 7 & $\mathrm{CP}_{3}$ (triangular) & 972.4 & 1.1 & 2 \\
\hline 8 & $\mathrm{CP}_{4}$ (square) & 103.4 & 0.01 & 1 \\
\hline 9 & $\mathrm{CP}_{4}$ (square) & 211.8 & 0.8 & 2 \\
\hline 10 & $\mathrm{CP}_{4}$ (square) & 272.6 & 0.02 & 1 \\
\hline 11 & $\mathrm{CP}_{4}$ (square) & 428.3 & 6.5 & 2 \\
\hline 12 & $\mathrm{CP}_{4}$ (square) & 432.4 & 1.1 & 1 \\
\hline 13 & $\mathrm{CP}_{4}$ (square) & 466.6 & 0.01 & 1 \\
\hline 14 & $\mathrm{CP}_{4}$ (square) & 568.9 & 6.2 & 1 \\
\hline
\end{tabular}

\begin{tabular}{|c|c|c|c|c|}
\hline \multicolumn{5}{|c|}{$\mathrm{C}_{\mathrm{n}} \mathrm{P}$ clusters } \\
\hline 1 & $\mathrm{C}_{2} \mathrm{P}$ (linear) & 200.0 & 4.8 & 1 \\
\hline 2 & $\mathrm{C}_{2} \mathrm{P}$ (linear) & 542.4 & 0.01 & 1 \\
\hline 3 & $\mathrm{C}_{2} \mathrm{P}$ (linear) & 563.9 & 80.1 & 1 \\
\hline 4 & $\mathrm{C}_{2} \mathrm{P}$ (triangular) & 649.0 & 5.0 & 1 \\
\hline 5 & $\mathrm{C}_{2} \mathrm{P}$ (triangular) & 672.6 & 2.0 & 1 \\
\hline 6 & $\mathrm{C}_{2} \mathrm{P}$ (triangular) & 1422.0 & 17.0 & 1 \\
\hline 7 & $\mathrm{C}_{3} \mathrm{P}$ (pyramidal) & 421.5 & 39.8 & 1 \\
\hline 8 & $\mathrm{C}_{3} \mathrm{P}$ (pyramidal) & 479.3 & 3.5 & 1 \\
\hline 9 & $\mathrm{C}_{3} \mathrm{P}$ (pyramidal) & 661.1 & 46.7 & 1 \\
\hline 10 & $\mathrm{C}_{3} \mathrm{P}$ (pyramidal) & 995.9 & 31.2 & 1 \\
\hline 11 & $\mathrm{C}_{3} \mathrm{P}$ (pyramidal) & 1381.6 & 17.5 & 1 \\
\hline 12 & $\mathrm{C}_{3} \mathrm{P}$ (triangular) & 189.1 & 3.0 & 2 \\
\hline 13 & $\mathrm{C}_{3} \mathrm{P}$ (triangular) & 349.0 & 21.6 & 1 \\
\hline 14 & $\mathrm{C}_{3} \mathrm{P}$ (triangular) & 603.9 & 0.01 & 1 \\
\hline 15 & $\mathrm{C}_{3} \mathrm{P}$ (triangular) & 668.2 & 22.2 & 2 \\
\hline \multicolumn{5}{|c|}{$\mathrm{C}_{\mathrm{n}} \mathrm{P}_{\mathrm{m}}$ clusters } \\
\hline 1 & $\mathrm{C}_{2} \mathrm{P}_{2}$ (rectangular) & 297.6 & 53.3 & 1 \\
\hline 2 & $\mathrm{C}_{2} \mathrm{P}_{2}$ (rectangular) & 468.9 & 0.01 & 1 \\
\hline 3 & $\mathrm{C}_{2} \mathrm{P}_{2}$ (rectangular) & 742.7 & 0.01 & 1 \\
\hline 4 & $\mathrm{C}_{2} \mathrm{P}_{2}$ (rectangular) & 792.7 & 148.0 & 1 \\
\hline 5 & $\mathrm{C}_{2} \mathrm{P}_{2}$ (rectangular) & 962.7 & 0.02 & 1 \\
\hline 6 & $\mathrm{C}_{2} \mathrm{P}_{3}$ (linear) & 44.2 & 3.7 & 1 \\
\hline 7 & $\mathrm{C}_{2} \mathrm{P}_{3}$ (linear) & 111.0 & 4.3 & 1 \\
\hline 8 & $\mathrm{C}_{2} \mathrm{P}_{3}$ (linear) & 148.2 & 6.3 & 1 \\
\hline 9 & $\mathrm{C}_{2} \mathrm{P}_{3}$ (linear) & 223.4 & 0.8 & 1 \\
\hline 10 & $\mathrm{C}_{2} \mathrm{P}_{3}$ (linear) & 338.9 & 14.8 & 1 \\
\hline 11 & $\mathrm{C}_{2} \mathrm{P}_{3}$ (linear) & 381.1 & 9.4 & 1 \\
\hline 12 & $\mathrm{C}_{2} \mathrm{P}_{3}$ (linear) & 647.3 & 11.9 & 1 \\
\hline \multirow[t]{2}{*}{13} & $\mathrm{C}_{2} \mathrm{P}_{3}$ (linear) & 718.0 & 27.8 & 1 \\
\hline & $\mathrm{C}_{2} \mathrm{P}_{3}$ (linear) & 1787.3 & 311.3 & 1 \\
\hline 14 & $\mathrm{C}_{2} \mathrm{P}_{3}$ (bipyramidal) & 200.5 & 16.7 & 2 \\
\hline 15 & $\mathrm{C}_{2} \mathrm{P}_{3}$ (bipyramidal) & 330.1 & 26.5 & 1 \\
\hline 16 & $\mathrm{C}_{2} \mathrm{P}_{3}$ (bipyramidal) & 446.6 & 0.01 & 1 \\
\hline 17 & $\mathrm{C}_{2} \mathrm{P}_{3}$ (bipyramidal) & 644.3 & 0.5 & 2 \\
\hline 18 & $\mathrm{C}_{2} \mathrm{P}_{3}$ (bipyramidal) & 690.8 & 0.01 & 1 \\
\hline 19 & $\mathrm{C}_{2} \mathrm{P}_{3}$ (bipyramidal) & 771.5 & 16.2 & 2 \\
\hline 20 & $\mathrm{C}_{3} \mathrm{P}_{2}$ (bipyramidal) & 128.8 & 30.1 & 1 \\
\hline 21 & $\mathrm{C}_{3} \mathrm{P}_{2}$ (bipyramidal) & 272.7 & 3.1 & 1 \\
\hline 22 & $\mathrm{C}_{3} \mathrm{P}_{2}$ (bipyramidal) & 307.5 & 11.5 & 1 \\
\hline 23 & $\mathrm{C}_{3} \mathrm{P}_{2}$ (bipyramidal) & 349.5 & 9.5 & 1 \\
\hline 24 & $\mathrm{C}_{3} \mathrm{P}_{2}$ (bipyramidal) & 461.0 & 2.0 & 1 \\
\hline 25 & $\mathrm{C}_{3} \mathrm{P}_{2}$ (bipyramidal) & 628.1 & 9.4 & 1 \\
\hline 26 & $\mathrm{C}_{3} \mathrm{P}_{2}$ (bipyramidal) & 679.9 & 4.4 & 1 \\
\hline 27 & $\mathrm{C}_{3} \mathrm{P}_{2}$ (bipyramidal) & 976.5 & 25.1 & 1 \\
\hline 28 & $\mathrm{C}_{3} \mathrm{P}_{2}$ (bipyramidal) & 978.4 & 0.4 & 1 \\
\hline 29 & $\mathrm{C}_{3} \mathrm{P}_{3}$ (ring) & 281.0 & 0.01 & 2 \\
\hline 30 & $\mathrm{C}_{3} \mathrm{P}_{3}$ (ring) & 385.4 & 51.4 & 1 \\
\hline 31 & $\mathrm{C}_{3} \mathrm{P}_{3}$ (ring) & 386.7 & 72.3 & 2 \\
\hline 32 & $\mathrm{C}_{3} \mathrm{P}_{3}$ (ring) & 485.1 & 9.8 & 2 \\
\hline 33 & $\mathrm{C}_{3} \mathrm{P}_{3}$ (ring) & 490.7 & 0.01 & 1 \\
\hline 34 & $\mathrm{C}_{3} \mathrm{P}_{3}$ (ring) & 788.3 & 0.02 & 1 \\
\hline 35 & $\mathrm{C}_{3} \mathrm{P}_{3}$ (ring) & 861.6 & 0.01 & 1 \\
\hline 36 & $\mathrm{C}_{3} \mathrm{P}_{3}$ (ring) & 1126.5 & 76.4 & 2 \\
\hline 37 & $\mathrm{C}_{3} \mathrm{P}_{2}$ (linear) & 218.9 & 18.1 & 1 \\
\hline 38 & $\mathrm{C}_{3} \mathrm{P}_{2}$ (linear) & 247.7 & 0.2 & 1 \\
\hline 39 & $\mathrm{C}_{3} \mathrm{P}_{2}$ (linear) & 299.0 & 0.01 & 1 \\
\hline 40 & $\mathrm{C}_{3} \mathrm{P}_{2}$ (linear) & 339.8 & 0.03 & 1 \\
\hline 41 & $\mathrm{C}_{3} \mathrm{P}_{2}$ (linear) & 353.1 & 1.2 & 1 \\
\hline 42 & $\mathrm{C}_{3} \mathrm{P}_{2}$ (linear) & 503.8 & 4.9 & 1 \\
\hline 44 & $\mathrm{C}_{3} \mathrm{P}_{2}$ (linear) & 709.2 & 1.4 & 1 \\
\hline 45 & $\mathrm{C}_{3} \mathrm{P}_{2}$ (linear) & 710.1 & 172.8 & 1 \\
\hline 46 & $\mathrm{C}_{3} \mathrm{P}_{2}$ (bipyramidal) & 324.9 & 0.9 & 1 \\
\hline 47 & $\mathrm{C}_{3} \mathrm{P}_{2}$ (bipyramidal) & 524.5 & 12.3 & 1 \\
\hline 48 & $\mathrm{C}_{3} \mathrm{P}_{2}$ (bipyramidal) & 529.5 & 0.2 & 1 \\
\hline 49 & $\mathrm{C}_{3} \mathrm{P}_{2}$ (bipyramidal) & 624.9 & 0.09 & 1 \\
\hline 50 & $\mathrm{C}_{3} \mathrm{P}_{2}$ (bipyramidal) & 724.1 & 8.2 & 1 \\
\hline
\end{tabular}




\begin{tabular}{ccccc}
\hline 51 & $\mathrm{C}_{3} \mathrm{P}_{2}$ (bipyramidal) & 775.5 & 45.7 & 1 \\
52 & $\mathrm{C}_{3} \mathrm{P}_{2}$ (bipyramidal) & 947.6 & 220.2 & 1 \\
53 & $\mathrm{C}_{3} \mathrm{P}_{2}$ (bipyramidal) & 1130.7 & 2.1 & 1 \\
\hline
\end{tabular}

$\mathbf{C}_{\mathbf{n}} \mathbf{P}$ clusters $\left(\mathbf{C}_{2} \mathbf{P}, \mathbf{C}_{3} \mathbf{P}\right)$ : Formally adding a single $\mathrm{P}$ to the $\mathrm{C}_{2}$ molecule can produce a linear model of $\mathrm{C}_{2} \mathrm{P}$ [Fig. 2(v)] similar to that of $\mathrm{C}_{2} \mathrm{Al}$ in which $\mathrm{Al}$ sits at the center and two $\mathrm{C}$ at the two sides. The CP bond length is found to be $1.870 \AA$. In the triangular model $\mathrm{C}_{2} \mathrm{P}$ [Fig. 2(vi)] the $\mathrm{CP}$ distance is $186.2 \AA$ and C-C distance $1.342 \AA$. The pyramidal model $\mathrm{C}_{3} \mathrm{P}$ [Fig. 2(vii)] is obtained by three carbon atoms which form a triangle and one $\mathrm{P}$ atom which sits at the top position. The $\mathrm{C}-\mathrm{P}$ bond distance is $1.925 \AA$ and the $\mathrm{C}-\mathrm{C}$ bond length is 1.462 $\AA$. While in $\mathrm{C}_{3} \mathrm{P}$ [Fig. 2(viii)] which is triangular, the three carbon atoms form a triangle and one phosphorus atom sits in the center. In this case the C-P bond length is $1.828 \AA$. The calculated vibrational frequencies are given in Table-2.

$\mathbf{C}_{\mathbf{n}} \mathbf{P}_{\mathrm{m}}$ clusters $\left(\mathbf{C}_{2} \mathbf{P}_{2}, \mathbf{C}_{2} \mathbf{P}_{3}, \mathbf{C}_{\mathbf{3}} \mathbf{P}_{2}\right.$ and $\left.\mathbf{C}_{3} \mathbf{P}_{3}\right)$ : In $\mathrm{C}_{2} \mathrm{P}_{2}$ which is rectangular [Fig. 2(ix)] all the atoms are alternatively arranged with bond length $1.870 \AA$ and in the linear $\mathrm{C}_{2} \mathrm{P}_{3}$ [Fig. 2(x)] $\mathrm{P}$ and $\mathrm{C}$ are located at the ends and two $\mathrm{P}$ and $\mathrm{C}$ lies in the center of the cluster. Interestingly the structure showed no linearity under optimization. The CP bond length is $1.6982 \AA$ and $\mathrm{C}-\mathrm{C}$ bond length is $1.2900 \AA$. Another $\mathrm{C}_{2} \mathrm{P}_{3}$ [Fig. 2(xi)] bipyramidal structure was obtained where the three phosphorus atoms form a triangle and one $\mathrm{C}$ atom sits on top position and another at the bottom position. The $\mathrm{CP}$ distance is found to be $1.913 \AA$ and the P-P distance 2.901 $\AA$. In $\mathrm{C}_{3} \mathrm{P}_{2}$ [Fig. 2(xii)] which is linear, three $\mathrm{C}$ atoms lie at the center and two $\mathrm{P}$ atoms at the ends. The CP distance at the ends is $1.870 \AA$. The bipyramidal $\mathrm{C}_{3} \mathrm{P}_{2}$ cluster [Fig. 2(xiii)] is obtained by three carbon atoms which form a triangle and a $\mathrm{P}$ atom which is at the top position and another in the bottom position. The CP distance is $2.295 \AA$ and the P-P distance is $3.196 \AA$. Another $\mathrm{C}_{3} \mathrm{P}_{3}$ structure [Fig. 2(xiv)] forms a hexagonal ring with alternate sites occupied by $\mathrm{C}$ and $\mathrm{P}$ atoms. All the vibrational frequencies are given in Table-2.

Energies of various clusters: A perusal of Table- 3 presents the zero point energies and total energies of the $\mathrm{C}_{\mathrm{n}} \mathrm{Al}_{\mathrm{m}}$ and $\mathrm{C}_{\mathrm{n}} \mathrm{P}_{\mathrm{m}}$ clusters. These values are graphically represented in Fig. 3 , which show that the total energies increase with increase in the number of cluster atoms. The sequence of the total energies of the cluster model $\mathrm{C}_{\mathrm{n}} \mathrm{Al}_{\mathrm{m}}$ is as follows: $\mathrm{CAl}(\mathrm{L}), \mathrm{C}_{2} \mathrm{Al}$ (L) $\mathrm{C}_{2} \mathrm{Al}_{2}(\mathrm{~L}), \mathrm{C}_{2} \mathrm{Al}$ (T), $\mathrm{C}_{3} \mathrm{Al}(\mathrm{T})<\mathrm{C}_{2} \mathrm{Al}_{2}(\mathrm{R}), \mathrm{C}_{3} \mathrm{Al}_{2}(\mathrm{~L}), \mathrm{C}_{3} \mathrm{Al}_{2}$ (BP) $\mathrm{C}_{2} \mathrm{Al}_{3}(\mathrm{~L})<\mathrm{C}_{2} \mathrm{Al}_{3}(\mathrm{BP}), \mathrm{C}_{3} \mathrm{Al}_{3}(\mathrm{R}), \mathrm{CAl}_{4}(\mathrm{~S})$. A comparison with the neighboring heteroatom-containing species that is $\mathrm{C}_{\mathrm{n}} \mathrm{P}_{\mathrm{m}}$ clusters have also been predicted. For each set $\{----\}$ where each member of form $\mathrm{C}_{\mathrm{n}} \mathrm{P}_{\mathrm{m}}$ is an increasing sequence in the total energy, we can further distinguish the groups of sets energetically as follows: $\left\{\mathrm{CP}(\mathrm{L}), \mathrm{C}_{2} \mathrm{P}(\mathrm{L}), \mathrm{C}_{2} \mathrm{P}(\mathrm{T}), \mathrm{C}_{3} \mathrm{P}(\mathrm{T})\right.$, $\left.\mathrm{C}_{3} \mathrm{P}(\mathrm{P}), \mathrm{C}_{2} \mathrm{P}(\mathrm{L})\right\}<\left\{\mathrm{C}_{2} \mathrm{P}_{2}(\mathrm{RA}), \mathrm{C}_{3} \mathrm{P}_{2}(\mathrm{~L}), \mathrm{C}_{3} \mathrm{P}_{2}(\mathrm{BP})\right\}<\left\{\mathrm{CP}_{3}\right.$ $\left.(\mathrm{T}), \mathrm{C}_{2} \mathrm{P}_{3}(\mathrm{~L}), \mathrm{C}_{2} \mathrm{P}_{3}(\mathrm{BP}), \mathrm{C}_{3} \mathrm{P}_{3}(\mathrm{R}), \mathrm{CP}_{4}(\mathrm{~S})\right\}$. This grouping suggests that in general (there are exceptions) linear and triangular isomers seems to have less energies than isomers of other symmetries. However, the comparison between the two $\mathrm{C}_{\mathrm{n}} \mathrm{Al}_{\mathrm{m}}$ and $\mathrm{C}_{\mathrm{n}} \mathrm{P}_{\mathrm{m}}$ in Fig. 3 suggest that $\mathrm{C}_{\mathrm{n}} \mathrm{Al}_{\mathrm{m}}$ isomers are usually less in energy than the $\mathrm{C}_{\mathrm{n}} \mathrm{P}_{\mathrm{m}}$ type. According to the results of our calculations, the less energy structures of the cluster models of $\mathrm{C}_{\mathrm{n}} \mathrm{Al}_{\mathrm{m}}$ and $\mathrm{C}_{\mathrm{n}} \mathrm{P}_{\mathrm{m}}$ can be regarded as the corresponding outcomes

\begin{tabular}{|c|c|c|c|}
\hline \multicolumn{4}{|c|}{$\begin{array}{c}\text { TABLE-3 } \\
\text { ZERO POINT VIBRATIONAL ENERGIES (ZPE) AND } \\
\text { TOTAL ENERGIES (TE) OF VARIOUS CLUSTERS }\end{array}$} \\
\hline $\begin{array}{l}\text { S. } \\
\text { No. }\end{array}$ & Cluster & $\begin{array}{l}\text { Zero point vibrational } \\
\text { energies }(\mathrm{eV})\end{array}$ & $\begin{array}{l}\text { Total energies } \\
(\mathrm{eV})\end{array}$ \\
\hline \multicolumn{4}{|c|}{$\mathrm{C}_{\mathrm{n}} \mathrm{Al}_{\mathrm{m}}$ cluster } \\
\hline 1 & $\mathrm{CAl}$ (linear) & 0.0465 & -278.8127 \\
\hline 2 & $\mathrm{CAl}_{2}$ (linear) & 0.0980 & -520.3326 \\
\hline 3 & $\mathrm{CAl}_{3}$ (triangular) & 0.1577 & -716.7942 \\
\hline 4 & $\mathrm{CAl}_{4}$ (square) & 0.1766 & -1003.2028 \\
\hline 5 & $\mathrm{C}_{2} \mathrm{Al}$ (linear) & 0.0614 & -316.3943 \\
\hline 6 & $\mathrm{C}_{2} \mathrm{Al}$ (triangular) & 0.1558 & -316.6618 \\
\hline 7 & $\mathrm{C}_{2} \mathrm{Al}_{2}$ (rectangular) & 0.1559 & -558.0945 \\
\hline 8 & $\mathrm{C}_{2} \mathrm{Al}_{3}$ (linear) & 0.2463 & -799.4108 \\
\hline 9 & $\mathrm{C}_{2} \mathrm{Al}_{3}$ (bipyramidal) & 0.2466 & -799.3859 \\
\hline 10 & $\mathrm{C}_{3} \mathrm{Al}$ (pyramidal) & 0.2771 & -354.3275 \\
\hline 11 & $\mathrm{C}_{3} \mathrm{Al}$ (triangular) & 0.1485 & -353.9456 \\
\hline 12 & $\mathrm{C}_{3} \mathrm{Al}_{2}$ (linear) & 0.3281 & -595.7709 \\
\hline 13 & $\mathrm{C}_{3} \mathrm{Al}_{2}$ (bipyramidal) & 0.2641 & -595.7200 \\
\hline 14 & $\mathrm{C}_{3} \mathrm{Al}_{3}$ (ring) & 0.2068 & -837.0267 \\
\hline \multicolumn{4}{|c|}{$\mathrm{C}_{\mathrm{n}} \mathrm{P}_{\mathrm{m}}$ cluster } \\
\hline 1 & $\mathrm{CP}$ (linear) & 0.0722 & -377.6220 \\
\hline 2 & $\mathrm{CP}_{2}$ (linear) & 0.1796 & -717.7843 \\
\hline 3 & $\mathrm{CP}_{3}$ (triangular) & 0.2164 & -1057.8079 \\
\hline 4 & $\mathrm{CP}_{4}$ (square) & 0.2542 & -1397.8502 \\
\hline 5 & $\mathrm{C}_{2} \mathrm{P}$ (linear) & 0.0809 & -415.1766 \\
\hline 6 & $\mathrm{C}_{2} \mathrm{P}$ (triangular) & 0.1700 & -415.2982 \\
\hline 7 & $\mathrm{C}_{2} \mathrm{P}_{2}$ (rectangular) & 0.2023 & -755.3707 \\
\hline 8 & $\mathrm{C}_{2} \mathrm{P}_{3}$ (linear) & 0.2727 & -1095.4947 \\
\hline 9 & $\mathrm{C}_{2} \mathrm{P}_{3}$ (bipyramidal) & 0.2913 & -1095.4931 \\
\hline 10 & $\mathrm{C}_{3} \mathrm{P}$ (pyramidal) & 0.2442 & -452.8670 \\
\hline 11 & $\mathrm{C}_{3} \mathrm{P}$ (triangular) & 0.1653 & -452.6463 \\
\hline 12 & $\mathrm{C}_{3} \mathrm{P}_{2}$ (linear) & 0.2096 & -793.0214 \\
\hline 13 & $\mathrm{C}_{3} \mathrm{P}_{2}$ (bipyramidal) & 0.3464 & -792.9778 \\
\hline 14 & $\mathrm{C}_{3} \mathrm{P}_{3}$ (ring) & 0.4392 & -1133.1905 \\
\hline
\end{tabular}

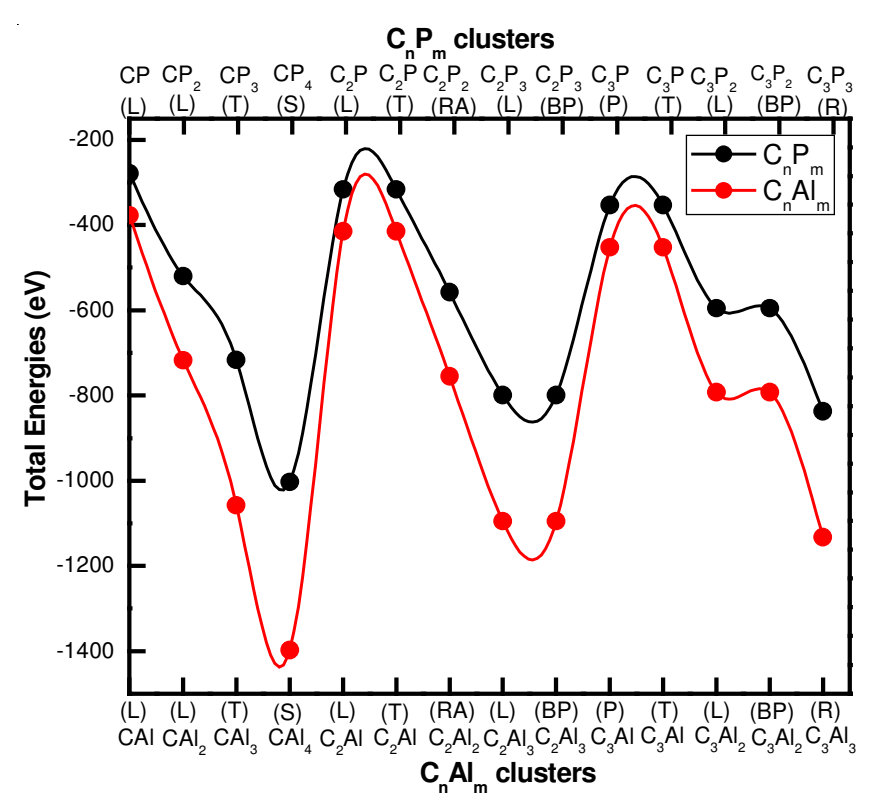

Fig. 3. Curves of total energies of the $\mathrm{C}_{n} \mathrm{Al}_{\mathrm{m}}$ and $\mathrm{C}_{\mathrm{n}} \mathrm{P}_{\mathrm{m}}$ clusters verses number of cluster atoms of different geometries L (linear), $\mathrm{T}$ (triangular), S (square), RA (rectangular), P (pyramidal), BP (bipyramidal) and $\mathrm{R}$ (ring)

of lengthening the carbon chain or the component atoms in the cluster models. As expected, the results obtained in our study for the two different types of cluster systems show that 
the values of the total energies varies and increases as the number of atoms in the isomers increases from $\mathrm{CAl}$ to $\mathrm{C}_{\mathrm{n}} \mathrm{Al}_{\mathrm{m}}$ and from $\mathrm{CP}$ to $\mathrm{C}_{\mathrm{n}} \mathrm{P}_{\mathrm{m}}$.

\section{Conclusion}

Density functional computations have been carried out on aluminum and phosphorous doped carbon clusters. Interesting results have been obtained, for example, comparison of frequencies of $\mathrm{CAl}_{3}$ (triangular) with $\mathrm{C}_{3} \mathrm{Al}$ (triangular) shows that $\mathrm{CAl}_{3}$ has a vibrational frequency $210.3 \mathrm{~cm}^{-1}$ whereas $\mathrm{C}_{3} \mathrm{Al}$ has a vibrational frequency $422.6 \mathrm{~cm}^{-1}$. Comparing $\mathrm{C}_{3} \mathrm{P}$ (triangular) with $\mathrm{CP}_{3}$ (triangular) we observe that shows that $\mathrm{CP}_{3}$ has vibrational frequency $222.8 \mathrm{~cm}^{-1}$ whereas $\mathrm{C}_{3} \mathrm{P}$ has vibrational frequency $189.1 \mathrm{~cm}^{-1}$ as the lowest frequency. It is observed that the effect of force constant is much stronger than the effect of the mass and our calculations are clear about the bond distance, which depends on the structures of various clusters. The sequences of the total energies of various isomers were obtained. Considerable effect due to the number of hetero atoms linked to carbon has been observed. We hope that the present work will serve to stimulate experimental studies of binary aluminum and phosphorus doped carbon clusters.

\section{REFERENCES}

1. F.Y. Naumkin, J. Phys. Chem. A, 112, 4660 (2008).

2. L. Zhang, C.B. Zhang and Y. Qi, Phys. Lett. A, 372, 2874 (2008).
3. H. Xing, S. Xu, Z. Ding, Y. Huang, X. Chen, J. Wang and Y. Shi, Phys. Lett. A, 372, 4694 (2008).

4. J.N. Ding, C.L. Li, N.Y. Yuan, G.Q. Ding, X.S. Chen, W. Luc, G.G. Chen and C.L. Chen, Phys. Lett. A, 374, 842 (2010).

5. A.N. Rosli, N.A. Zabidi, H.A. Kassim and K.N. Shrivastava, J. Clust. Sci., 21, 197 (2010).

6. C.C. Zhan and S. Iwata, J. Chem. Phys., 107, 7323 (1997).

7. G. Pascoli and H. Lavendy, J. Phys. Chem. A, 103, 3518 (1999).

8. G. Pascoli and H. Lavendy, Int. J. Mass Spectrom., 189, 125 (1999).

9. G. Pascoli and H. Lavendy, Int. J. Mass Spectrom., 206, 153 (2001).

10. Z.Y. Liu, R.B. Huang, Z.C. Tang and L.S. Zheng, Chem. Phys., 229, 335 (1998).

11. Z.Y. Liu, R.B. Huang and L.S. Zheng, Chem. J. Chin. Univ., 18, 2019 (1997).

12. K. Fisher, I. Dance and G. Willett, Eur. Mass Spectrom., 3, 331 (1997).

13. R. Zeng, J.B. Liu, C.Y. Hang and Z. Gao, Chem. J. Chin. Univ., 21, $581(2000)$.

14. E. Delrio, C. Barrientos and A. Largo, J. Phys. Chem., 100, 585 (1996).

15. A.I. Boldyrev, J. Simons, X. Li and L.S. Wang, J. Am. Chem. Soc., 121, 10193 (1999).

16. N.A. Cannon, A.L. Boldyrev, X. Li and L.S. Wang, J. Chem. Phys., 113, 2671 (2000).

17. X. Li, L.S. Wang, N.A. Cannon and A.I. Boldyrev, J. Chem. Phys., 116, 1330 (2002)

18. D. Lopez-Duran, M.P. De Lara-Castells, G. Delgado-Barrio, P. Villareal, C. Di Paola, F.A. Gianturca and J. Jellinek, Phys. Rev. Lett., 93, 053401 (2004). 